\title{
The Energy Spectrum of Imperfect Kronig-Penney Model
}

\author{
Abdurrouf, M.A. Pamungkas, M. Nurhuda
}

\begin{abstract}
In this work the influence of imperfections of the potential of Kronig-Penny on its energy spectrum is investigated. This model is solved by our newly developed filter method. Imperfections are modelled by removing some potential barriers or changing the width or height of the potential. For the imperfect Kronig-Penny model we observe some energy states in the band gap area. This result is very important for the investigation the properties of heterostructure material.
\end{abstract}

Keywords: Kronig-Penney; energy spectrum; filter method; imperfection; properties of heterostructure material.

\section{INTRODUCTION}

The fast development of technology requires the availability of materials with specific properties. To overcome, scientists should be able to understand, control, and manufacture materials with certain mechanical, electrical, optical, and magnetic properties. Since most material properties are strongly influenced by the energy band pattern, which is the solution to the Schrödinger equation for the material, the development of analytical and numerical techniques for solving the Schrödinger equation is an important contribution [1].

Finding an analytical solution for Schrödinger equation is not always a simple task. It depends on the potential form (including its dimension, its symmetry, its periodicity, and its linearity), its dependence on time, number of particles, and the existence of external filed. As an alternative, scientist prefer to use a numerical solution. Recently, we developed an alternative numerical method for solving the Schrödinger equation, known as the filter method [2]. The filter method has a virtue that the user can obtain the eigen energy and eigenfunction -in a specific energy range- of any given potential of interest, without defining boundary conditions. We have implemented the filter method to the Schrödinger equation for single particle in single potentials such as electron on Coulomb potential and electron in harmonic oscillator potential, with a great success. The method has also been implemented for multi particle systems, such as electrons in helium atoms, where the acceptable results can be reproduced with the precision until the $9^{\text {th }}$ decimal [3].

Another attractive challenge is the implementation of filter method for periodic potential, such us Kronig-Penney potential. The Kronig-Penney potential is actually a periodic

Revised Manuscript Received on January 10, 2020.

* Correspondence Author

Abdurrouf*, Physics Department, Universitas Brawijaya, Malang, Indonesia. Email: abdurrouf@ub.ac.id

Mauludi Ariesto Pamungkas, Physics Department, Universitas Brawijaya, Malang, Indonesia. Email: m_ariesto@ub.ac.id

Muhammad Nurhuda, Proffesor, Physics Department, Universitas Brawijaya, Malang, Indonesia. Email: mnurhuda@ub.ac.id form of a combination of limited-height potential barriers and potential wells [4]. The analytic solution of the Kronig-Penney potential has been studied and it is well known [5]. In meanwhile, the numerical solution was investigated by various methods, such as vibrational method] [8], matrix method $[9,10]$ and tight binding formulation [11]. We have implemented the filter method for single particle subject to the one-dimensional periodic potential, such as oscillator potential and Kronig-Penney potential, where we obtain energy band structure [12-13]. The band structure for Kronig-Penney potential has been compared with the analytical solution, where they match to each other until the $2^{\text {nd }}$ decimal [13].

Later, the Kronig-Penny model was used to model two-layer graphene [14] and multiple quantum well and supperlattice solar cells [15-17]. In addition, the effect of impurities [18-19] and the effect of external fields [20] on Kronig-Penney model are also very attractive topic. For this case, analytical solution become more complicated. Therefore, it is interested to implement the filter method. This article aims to implement a filter method for the imperfect Kronig-Penney potential, to obtain the energy spectrum, and to analyze the results. This result is very important for the investigation the properties of heterostructure material.

\section{MATERIALS AND METHODS}

The Kronig-Penney model is a simplified model for an electron in a one-dimensional periodic potential. The model consists of an infinite periodic array of rectangular potential barriers and potential well, as depicted in Figure 1. This model has an advantage that it enables us to analytically determine the eigenvalues and eigenfunctions. It is also possible to obtain analytic expressions for the dispersion relation and the electron density of states.

Consider a Kronig-Penney model with rectangular potential's width of $a$, potential well's width of $b$, and potential barrier's height of $V_{0}$, then the eigenvalues of energy is given by transcendental formula [5]:

$$
\begin{aligned}
\left(\frac{Q^{2}-K^{2}}{2 Q K}\right) \sinh Q a \sin K b \\
+\cosh Q a \cos K b=\cos k(a+b)
\end{aligned}
$$

In above, $K=\frac{\sqrt{2 m E}}{\hbar}$ and $Q=\frac{\sqrt{2 m\left(V_{0}-E\right)}}{\hbar}$, where $E$ is energy of electron. It is also possible to obtain the dispersion relation $(E \quad$ vs. $k)$ from Equation (1). 


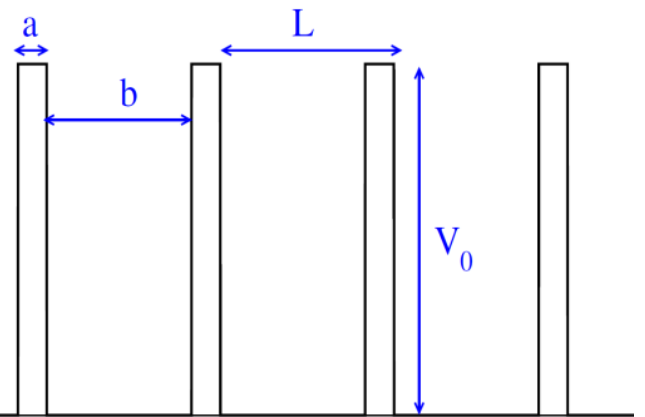

Fig. 1. Potential of Kronig-Penney. The width of rectangular potential barrier is $a$, the width of potential well is $b$, and the potential height is $V_{0}$. It is easy to know that the width of lattice is $L=a+b$ and the width ratio of potential barrier to potential well is $r=a / b$.

Despite the existence of an analytical solution to the perfect Kronig-Penney model, it is difficult to analytically determine the solution for the imperfect model or the model under external field. The imperfection in Kronig-Penney model can be obtained by (i) changing the height of some potential barriers, (ii) changing the width of some potential barriers, (iii) changing the depth of some potential wells, (iv) changing the width of some potential wells, (v) removing some potential barrier, (vi) removing some potential wells, or (vii) a combination thereof. For those case, it is very challenging to use the filter method.

The idea behind the filter method is the concept of wave's superposition, where any arbitrary wave packet is actually a superposition of all possible eigenfunction. To select the specific eigenfunction from a given wave packet, one need to apply the appropriate operator to the wave packet. To this end, we define on operator

$$
\widehat{F}=\frac{1}{\hat{H}-E_{n}},
$$

Where $F$ and $H$ stands for filter and Hamiltonian operator respectively. In Equation (2), $E_{n}$ is the eigen-energy, whose corresponding eigenfunction will be selected. Once operator $F$ applied to the wavefunction, only the selected eigenfunction with $E=E_{n}$ grows up, while other eigenfunctions whose energies are not matched, decreases. Mathematically, it can be written as follow.

$$
\begin{aligned}
\hat{F} \psi(\mathrm{r}) & =\frac{1}{\widehat{H}-E_{n}} \sum_{m} a_{m} \phi_{m}(\mathrm{r}) \\
& =\sum_{m} a_{m} \phi_{m}(\mathrm{r}) \delta\left(E_{m}-E_{n}\right) \\
& \widetilde{\phi}_{n}^{1}(\mathrm{r})
\end{aligned}
$$

However the eigenfunction $\tilde{\phi}_{n}^{1}(\mathrm{r})$ is not been normalized and therefore should be normalized as follow

$$
\phi_{n}^{1}(\mathrm{r})=\frac{\tilde{\phi}_{n}^{1}(\mathrm{r})}{\sqrt{d^{3} \mathrm{r}\left|\tilde{\phi}_{n}^{1}(\mathrm{r})\right|^{2}}}
$$

Next, by applying Hamiltonian operator on the normalized eigenfunction, we can calculate the eigen energy as

$$
E_{n}^{1}=\int d^{3} \mathrm{r} \phi_{n}^{1}(\mathrm{r}) \hat{H} \phi_{n}^{1}(\mathrm{r}) .
$$

In implementation, this process can be repeated for a range of energy to obtain the eigen energy and egeinfunction in that region. In solving Schroedinger equation, we use Hartree atomic units with $e=m=\hbar=k=1$ and $\mathrm{c}=137$.
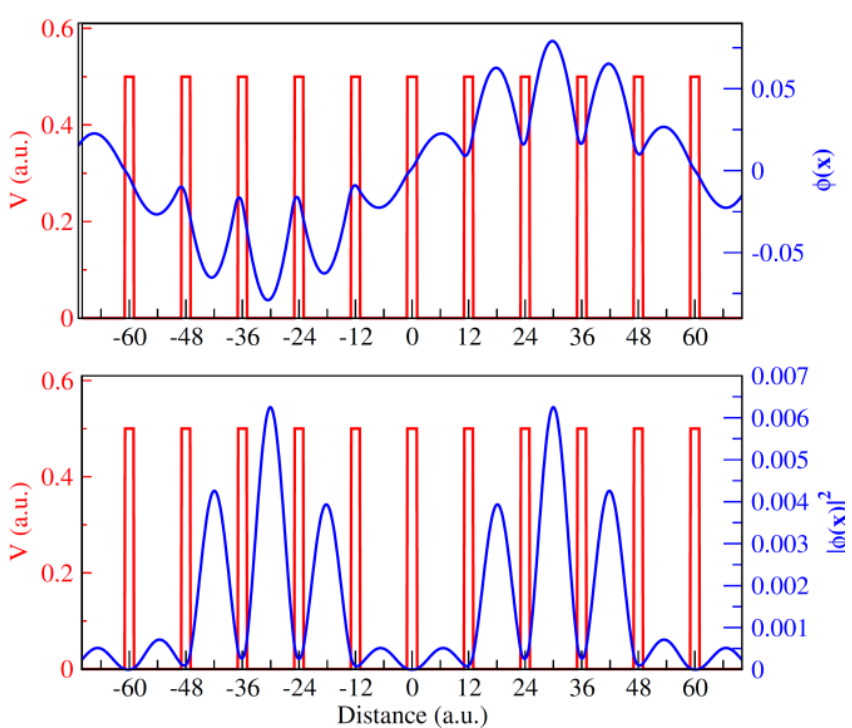

Fig. 2. The eigenfunctions of first energy level (blue curve, upper panel) and its modulus square (blue curve, lower panel) of perfect Kronig-Penney potential (red curve).

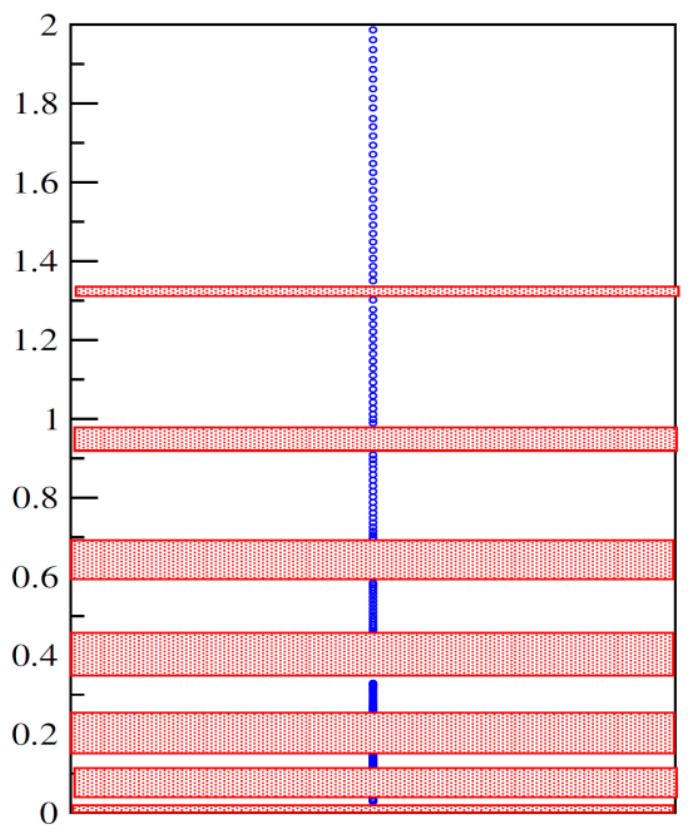

Fig. 3. The energy spectrum obtained by using filter method. The band gaps are marked by red colour.

\section{RESULTS AND DISCUSSION}

Let's first discuss one-dimensional perfect Kronig-Penney model. The simulation was carried out on a crystal consisting of 50 lattices. We use a lattice characterized by width of lattice $L=12$ a.u., width of barrier $b=2$ a.u., width of well $b=10 \mathrm{a} . \mathrm{u}$, and height of barrier $V_{0}$ $=0.5$ a.u. The integration in filter method is done along the crystal, from $\mathrm{x}=-300$ a.u. up to $x=300$ a.u. with $d x=0.1$ a.u., and for energy range of interest, i.e. from $E=0$ a.u. up to $E=2$ a.u. with $d E=0.001$ a.u. The first eigenfunction and its modulus square for perfect Kronig-Penney model are

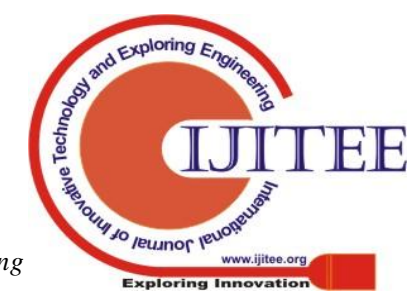


displayed in Figure 2. The Figure shows that the eigenfunction towards to zero in the barrier potential range, as expected.

In Figure 3, we display energy spectrum obtained numerically by using filter method. It was clear that the filter method list all allowed energy level, regardless to its wave number $k$. However, it is still possible to obtain the dispersion relation from filter method, by evaluating wave number $k$ from its eigenfunction. The important point to notice here is that both solutions draw the same conclusion, that for the energy range from 0 a.u. to 2 a.u. we obtain 7 energy bands (marked by white background) and 7 energy gabs (marked by red background). The analytical and numerical value of these energy bands and energy gabs matches to each other till the second decimals [13]. In spite of the disadvantage of filter method in finding dispersion relation, filter still works for imperfect Kronig-Penney potential, when the analytical solution need much modification.
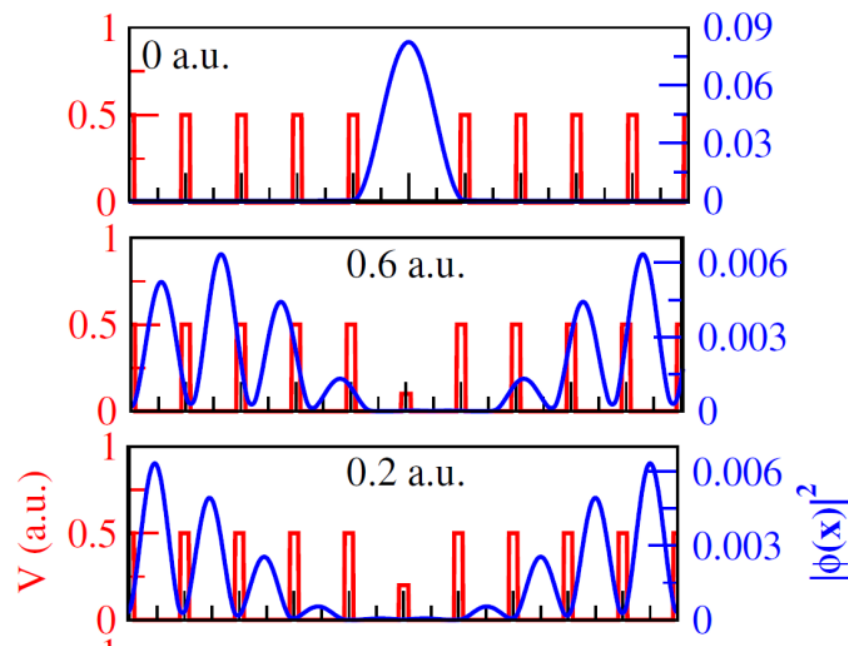

Fig. 4. The profile imperfect Kronig-Penney potential (red curve) and modulus square of the corresponding eigenfunctions (blue curve), ), for various height of the potential barrier in the center of crystal, $V_{C}<0.5$ a.u., which is located at position $x=0$ a.u.

Let's further investigate the imperfect Kronig-Penney model. We model the imperfection by modifying one of the potential barriers by changing its height. Of the 51 potential barriers in the crystal, we modify the potential barrier in the middle, which is located at position $x=0$ a.u. and vary their height from $V_{C}=0$ a.u. to $V_{C}=1$ a.u. with an increment of $\Delta V_{C}=1$ a.u. The potential profile and modulus square of the corresponding eigenfunctions are depicted in Figure 4-5. From the figure, we observe that the eigenfunction towards to

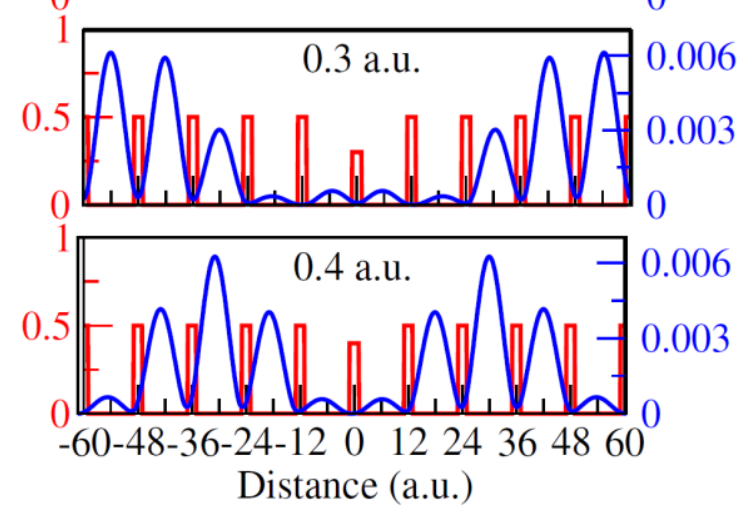

zero during the potential barrier. Therefore, by setting $V_{C}<0$ a.u. (upper panel of Fig. 4), we obtain a great modulus square of eiegenfunction in the center of crystal.

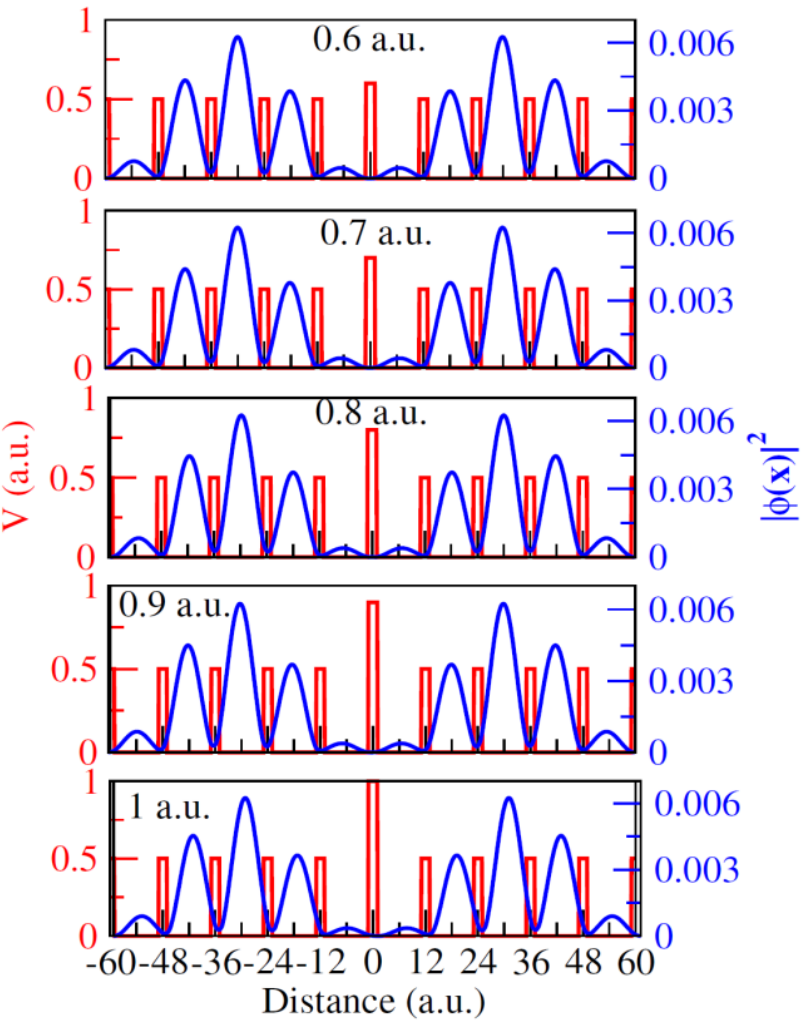

Fig. 5. The profile imperfect Kronig-Penney potential (red curve) and modulus square of the corresponding eigenfunctions (blue curve), for various height of the potential barrier in the center of crystal, $V_{C}>0.5$ a.u., which is located at position $x=0$ a.u..

The effect of imperfections on the energy spectrum is shown in Figure 6. It can be seen from Figure 6 that the imperfection has two different effects, i.e. imperfection due to reduction or enhancement of potential's height. Reduction potential's height $\left(V_{C}<0.5\right.$ a.u , Fig. 4$)$ causes the presence of energy levels in energy gabs range, just below energy band regime. For the lower potential $V_{C}$, the position of the existing energy go away from the energy band. Then, we have a shallow energy level for $V_{C} \leq 0.5$ a.u. and have a deep energy level for $V_{C}<<0.5$ a.u. For a fix $V_{C}$ the existing energy level towards to energy band for higher energy value. The enhancement in potential's height $\left(V_{C}>0.5\right.$ a.u. $)$ has different effects. It causes the presence of energy gabs in energy upper part of energy band regime. We notice the energy gap in the upper part of the $6^{\text {th }}$ energy band (just below $E=1.3$ a.u) for $V_{C} \geq 0.7$ a.u..

Imperfections can also be modeled by eliminating more than one potential. As we already discuss, removing a potential barrier leads to creation of an energy levels in the band gap regime. If we remove more potential barrier, the band gab regime will have more energy level and they will make a new energy band. In the case of high-energy bands, this new energy band merges with the old energy band and appears to be expanding. In the case of low-energy bands, the new energy band remains separate from the old energy

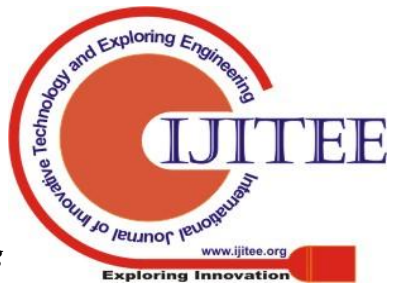


band. It is therefore easy to generalize that if we remove all potential barriers, we have free electrons with a continuous energy level.

We note here that the new energy level also can be obtained by changing the width and the depth of potential barrier. The fact that the filter method can handle the imperfect Kronig-Penney model, suggests its implementation as tool for analyzing multi quantum well and heterostructure material.

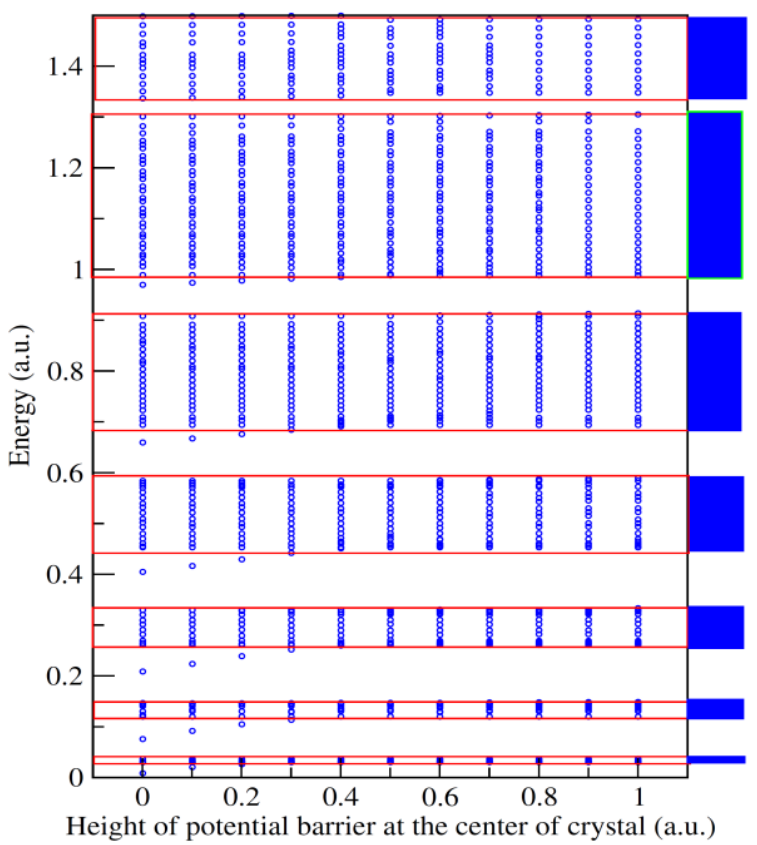

Fig. 6. The profile imperfect Kronig-Penney potential (red curve) and modulus square of the corresponding eigenfunctions (blue curve), for various height of the potential barrier in the center of crystal, $V_{C}$, which is located at position $x=0$ a.u.

\section{CONCLUSION}

To conclude, we have already implement the filter method for analyzing imperfect Kroning-Penny model. For this model, we observe the existence of new energy level and/or energy gabs in band gab regime.

\section{ACKNOWLEDGMENT}

The authors thank to DRPM Kemenristekdikti for financial support through Word Class Research, academic year 2019.

\section{REFERENCES}

1. Pamungkas, M. A. Setyowati, D. A. and Abdurrouf, Optical properties of Ga-doped silicene and as-doped silicene: First principle calculations, AIP Conference Proceedings, vol 2021, pp. 050014, 2018

2. Nurhuda, M. dan Rouf, A, Filter method without boundary-value condition for simultaneous Schroerdinger equation on a grid, Phys. Rev. E, Vol. 96 No. 3, pp. 033302(1-9), 2017

3. Nurhuda, M, Abdurrouf, and Pamungkas, M.A., Implementation of a filter method for simultaneous computation of eigenfunction and eigenenergy of a helium atom on grids, Phys. Rev. E (submitted), 2019

4. C. Kittel, Introduction to Solid State Physics, $8^{\text {th }}$ ed, John Wiley \& Sons Inc, pp. 168-169, 2005

5. D. A. McQuarrie, The Kronig-Penney model: A single lecture illustrating the band structure of solids, The Chemical Educator, Vol. No. 1, pp. 1-10, 1996
6. Alexander, S.A. and Coldwell, R.L. Solution of the Kronig-Penney model using variational Monte Carlo, Int. J. Quantum Chem, Vol. 86, pp. 325-329, 2002)

7. Mishra, S. and Satpathy, S., One-dimensional photonic crystal: The Kronig-Penney model, Phys. Rev. B, Vol. 68 No. 4, pp. 045121(9), 2003

8. A. Shuaibu and M. Y. Onimisi, Simulationof energy levels for electron subjected to 1d periodic potentials based on nearly free approximation, Am. J. Cond. Matt. Phys., Vol. 2, No. 3, pp. 63-66, 2012

9. F. Le Vot, J. J. Meléndez and S. B. Yuste, Numerical matrix method for quantum periodic potentials, Am. J. Phys, Vol. 84 No. 6, pp. 426, 2016

10. R. L. Pavelich and F. Marsiglio (2015), The Kronig-Penney model extended to arbitrary potentials via numerical matrix mechanics, Am. J. Phys, Vol. 83, pp. 773, 2016

11. F. Marsiglio and R. L. Pavelich, The tight-binding formulation of the Kronig-Penney model, Sci. Rep., Vol. 7, No. 1, pp, 17223(9), 2017

12. Abdurrouf, Nurhuda, M., and Wiyono, Modelling one-dimensional crystal by using harmonic oscillator potential, IOP Conf. Ser.: Mater. Sci. Eng., Vol. 546, pp. 052001(1-6), 2019.

13. Abdurrouf, Pamungkas, M.A., Wiyono, and Nurhuda, Implementation of filter method to solve the Kronig-Penney model, AIP Conf. Series (in Press), 2019

14. M. Barbier, P. Vasilopoulos, and F. M. Peeters, Kronig-Penney model on bilayer graphene: Spectrum and transmission periodic in the strength of the barriers, Phys. Rev. B Vol. 82 No. 23, 235408(10) 2010

15. Courel, M, Rimada, J.C. and Hernández, GaAs/GaInNAs quantum well and superlattice solar cell, Prog. Photovolt: Res. Appl., Vol. 21, pp. 276-282, 2013

16. Courel, M, Rimada, J.C. and Hernández, GaAs/GaInNAs quantum well and superlattice solar cell, Applied Physics Letters Vol. 100, pp. 073508, 2012.

17. Zhang, Y and Wang, Y, Energy Band Analysis of MQW Structure Based on Kronig-Penny Model, Journal of Modern Physics Vol. 4, pp. 968-973, 2013.

18. Engstedt, E, Quantum periodic potentials with impurities: The effect of impurities on the one-dimensional Kronig-Penney potential, School of Engineering Sciences, KTH Royal Institute, 2019.

19. Luna-Acosta, G.A, Schanze, H, Kuhl, U, and Stöckmann, H-J, Impurity effects on the band structure of onedimensional photonic crystals: experiment and theory, New Journal of Physics Vol. 10, pp. 043005(16) 2008

20. Faisal, F.H.M and Geneiser, R, Exact solution of the Kronig-Penney model of 1D crystal in strong laser fields, Physics Letters A Vol. 141 No. 5-6, pp. 297-300, 1989 .

\section{AUTHORS PROFILE}

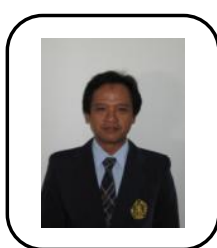

Abdurrouf, obtained his doctor's degree from Faculty of Physics, University of Bielefeld (Germany) with focus on the intense field theory of high harmonic generation from freely rotating molecules. His current resaerch focuses on computational molecules. Now, he is head of physics laboratory at physics department Universitas of Brawijaya, Indonesia.

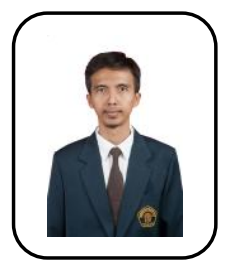

Mauludi Ariesto Pamungkas, obtained his doctor's degree from Universtiy of Science and Technology (Korea) in the field of Nanomaterials Science and Engineering. His current resaerch focuses on computational material. Now, he is head of simulation and modeling laboratory at physics department Universitas of Brawijaya, Indonesia.

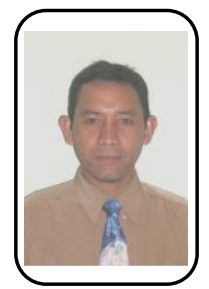

Nuhammad Nurhuda, obtained his doctor's degree from Faculty of Physics, University of Bielefeld (Germany) with focus on development of methods of time dependent simulations for atomic dynamics in intense laser field and application to chirp control of multiphoton processes. His current resaerch focuses on computational atoms and molecules. Now, he is head of physics department, Universitas of Brawijaya, Indonesia. He has published many research papers and become a referee in in many reputed journals. He also a member of many Physical Societies.

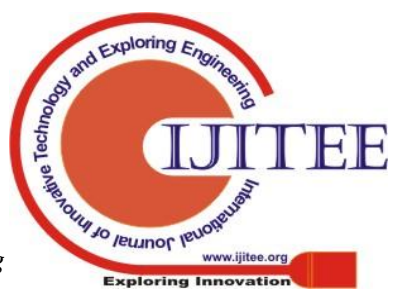

